\title{
Therapeutic hypothermia effect on asphyxial cardiac arrest-induced renal ischemia/reperfusion injury via change of Nrf2/HO-1 levels
}

\author{
ALI JAWAD ${ }^{1 *}$, YEO-JIN YOO ${ }^{1 *}$, JEONG-HWI CHO ${ }^{1}$, JAE CHOL YOON $^{2}$, WEISHUN TIAN ${ }^{1}$, \\ MOHAMMAD SADIKUL ISLAM ${ }^{1}$, EUI-YONG LEE ${ }^{1}$, HA-YOUNG SHIN ${ }^{1}$, SO EUN KIM ${ }^{2}$, KYUNGHWA KIM ${ }^{3}$, \\ DONGCHOON AHN ${ }^{1}$, BYUNG-YONG PARK $^{1}$, IN-SHIK KIM $^{1}$, JUN HO LEE $^{4}$ and HYUN-JIN TAE ${ }^{1}$ \\ ${ }^{1}$ Department of Veterinary Medicine and Bio-Safety Research Institute, Jeonbuk National University, Iksan, \\ Jeollabuk-do 54696; ${ }^{2}$ Department of Emergency Medicine, Research Institute of Clinical Medicine of \\ Jeonbuk National University; Departments of ${ }^{3}$ Thoracic and Cardiovascular Surgery and ${ }^{4}$ Anesthesiology and \\ Pain Medicine, Research Institute of Clinical Medicine of Jeonbuk National University-Biomedical Research Institute of \\ Jeonbuk National University Hospital, Jeonju, Jeollabuk-do 54907, Republic of Korea
}

Received February 26, 2021; Accepted June 16, 2021

DOI: $10.3892 / \mathrm{etm} .2021 .10463$

\begin{abstract}
The present study aimed to investigate the renoprotective effect of therapeutic hypothermia $(\mathrm{TH})$ on renal ischemia-reperfusion injury (RI/RI) induced by asphyxial cardiac arrest (CA) in rats. A total of 48 male rats were randomly divided into five groups: i) Sham $(n=6)$; ii) Normothermia + CA (Normo.) $(\mathrm{n}=14)$; iii) Normo. and $2 \mathrm{~h}$ of TH after return of spontaneous circulation (ROSC) $(n=12)$; iv) Normo. and $4 \mathrm{~h}$ of TH after ROSC $(n=9)$; and v) Normo. and $6 \mathrm{~h}$ of $\mathrm{TH}$ after ROSC $(\mathrm{n}=7)$. All rats except the Sham group underwent asphyxia $\mathrm{CA}$ and were sacrificed 1 day after ROSC. The survival rate increased from $42.8 \%$ in the Normo. group to $50,66.6$ and $85.7 \%$ in the groups with 2,4 and $6 \mathrm{~h}$ of $\mathrm{TH}$ after $\mathrm{CA}$, respectively. TH attenuated the histopathological changes of the renal tissues following ROSC and the levels of blood urea nitrogen, serum creatinine and malondialdehyde in
\end{abstract}

Correspondence to: Professor Hyun-Jin Tae, Department of Veterinary Medicine and Bio-Safety Research Institute, Jeonbuk National University, 79 Gobong-ro, Iksan, Jeollabuk-do 54696, Republic of Korea

E-mail: hjtae@jbnu.ac.kr

Dr Jun Ho Lee, Department of Anesthesiology and Pain Medicine, Research Institute of Clinical Medicine of Jeonbuk National University-Biomedical Research Institute of Jeonbuk National University Hospital, 20 Geonji-ro, Jeonju, Jeollabuk-do 54907, Republic of Korea

E-mail: gojuno@jbnu.ac.kr

*Contributed equally

Key words: cardiac arrest, cardiopulmonary resuscitation, therapeutic hypothermia, nuclear erythroid-2-related factor 2 , heme oxygenase 1 renal tissues. On immunohistochemistry, the relative optical density of nuclear erythroid-related factor-2 (Nrf2) and heme oxygenase (HO-1) expression in renal tissues increased in the Normo. group compared with that in the Sham group and exhibited further significant increases at $6 \mathrm{~h}$ of $\mathrm{TH}$ after ROSC. In conclusion, $\mathrm{TH}$ attenuated renal injury and increased the expression of Nrf2 and HO-1 in a $\mathrm{TH}$ treatment time-dependent manner.

\section{Introduction}

Cardiac arrest (CA) refers to loss of heart function that results in an abrupt halt of effective blood flow to the body; the morbidity and mortality due to CA have increased worldwide (1). The annual incidence of sudden CA (SCA) is $~ 3$ million; however, the survival rate of SCA is $<1 \%$ (2). Roberts et al (3) have reported that dysfunctions in various organs are common after CA following return of the spontaneous circulation (ROSC). In particular, kidney injury that results from CA following ROSC is a complex process; for heart disease patients admitted to hospitals, impairment of renal injury is common and is associated with a high mortality rate (4). Acute kidney injury (AKI) occurs frequently in patients with CA, occurring in $~ 50 \%$ of cases of CA (5). Geri et al (6) reported that the incidence of acute renal injury ranges from 12 to $40 \%$ in patients with CA. Most studies have focused on myocardial dysfunction and brain injury following ROSC after CA; however, renal injury has not been widely studied $(7,8)$.

Oxidative stress contributes to the pathogenicity of renal ischemia/reperfusion injury (RI/RI) (9). Furthermore, $\mathrm{RI} / \mathrm{RI}$-induced oxidative stress generates high levels of reactive oxygen species (ROS). Subsequently, overproduction of ROS results in mutation of DNA, apoptosis, necrosis and lipid peroxidation, causing cellular death in numerous ways $(10,11)$. A signaling pathway determined to have anti-oxidative stress properties and scavenge ROS production under oxidative stress conditions is nuclear erythroid-related factor-2 (Nrf2)/heme 
oxygenase (HO-1). Nrf2, an inducible transcription factor, binds with the antioxidant response element (ARE) located on the promoter regions of numerous antioxidant and detoxifying genes such as HO-1 (12). Previous studies $(13,14)$ mainly focused on histopathological and pathophysiological ailments and the expression levels of Nrf2 and HO-1 in rats induced by $\mathrm{RI} / \mathrm{RI}$; however, this type of study in a rat model of asphyxial $\mathrm{CA}$ is rare.

The first successful TH application in humans following ROSC after CA was reported near the end of the 1950s (15). Since then, TH has been the most successful treatment for CA (16). Mild TH (MTH) increases the survival rate and attenuates neurological outcomes in patients with $\mathrm{CA}$ who achieve ROSC (17). Furthermore, MTH protects the heart, liver and kidney from damage (18). Previous studies demonstrated that MTH provides damage protection against oxidative stress (18-20). However, other studies reported that the neurological outcome and survival rate following ROSC after CA were not significantly different from those of patients who did not achieve ROSC (21-23). As TH has a controversial effect in patients with $\mathrm{CA}$, an experiment was performed in the present study to confirm such an effect following ROSC.

Tujjar et al (24) demonstrated AKI in patients with CA following ROSC after CA, although the mechanism of kidney damage had remained elusive. Several studies reported increased expression of $\mathrm{Nrf} 2$ and $\mathrm{HO}-1$ in the RI/RI experimental animal model $(13,14)$. Thus, these parameters were selected for the present study to assess the antioxidative effects of TH in asphyxial CA-induced RI/RI following ROSC. The present study aimed to investigate the renoprotective effect of TH on asphyxial CA-induced RI/RI in rats.

\section{Materials and methods}

Experimental animals. Male Sprague-Dawley (SD) rats (total $\mathrm{n}=48$; bodyweight, 270-300 g; 10-weeks-old) were supplied by the Experimental Animal Center Jeonbuk National University (Jeonju, South Korea). They were housed in a conventional manner with adequate temperature $\left(23 \pm 2^{\circ} \mathrm{C}\right)$ and humidity $(60 \pm 10 \%)$ control under a 12-h light/dark cycle. They were provided with food and water ad libitum. All of the experimental procedures were approved by the Institutional Animal Care and Use Committee of Jeonbuk National University (approval no. JBNU 2019-005). Experimental animals were divided as into two major groups: Sham group (not subjected to CA surgery) $(n=6)$ and rats subjected to CA surgery (total $\mathrm{n}=42$ ). Rats that underwent CA surgery were further divided into the following groups: i) Normothermia + CA (Normo.) group $(n=14)$; ii) Normo. with 2 h TH immediately after ROSC and gradual increase of the temperature to the normal temperature until sacrifice (Hypo 2 h) $(n=12)$; iii) Normo. group with $4 \mathrm{~h}$ TH following cardiopulmonary resuscitation (CPR) and gradual rewarming to the normal temperature, which was maintained until the sacrifice of the rats (Hypo $4 \mathrm{~h}$ ) $(\mathrm{n}=9)$; iv) Normo. group with $6 \mathrm{~h} \mathrm{TH}$ immediately after ROSC and rewarming was performed to attain normal temperature, which was maintained until the rats were sacrificed (Hypo $6 \mathrm{~h})(\mathrm{n}=7)$. The number of rats in each group was selected according to the expected survival rate of each group with a target of having 6 animals per group at the end of the protocol; in contrast to the estimated high survival rate at 2 and $4 \mathrm{~h}$ of TH (25).

Induction of $C A$ and $C P R$. The induction of CA and CPR was performed according to the preferred protocol (26). In short, a rodent ventilator (Harvard Apparatus) was used to anesthetize the rats with $2-3 \%$ isoflurane and for mechanical ventilation. Peripheral oxygen saturation $\left(\mathrm{SpO}_{2}\right)$ was checked by connecting the pulse oximetry with the right leg. For electrocardiographic (ECG) assessment, ECG probes were inserted in the limbs and data were monitored regularly. The cannulation of the left femoral artery was for monitoring the mean arterial pressure (MAP) and the right femoral vein was for intravenous administration. Vecuronium bromide $(2 \mathrm{mg} / \mathrm{kg}$; Gensia Sicor Pharmaceuticals) was injected intravenously after a stabilization period of $5 \mathrm{~min}(27,28)$. Furthermore, mechanical ventilation was also stopped for the induction of asphyxial CA. The MAP reaching below $20 \mathrm{mmHg}$ resulting in pulseless electrical activity is defined as CA and it took 3-4 min for CA induction. After $5 \mathrm{~min}$ of CA with the administration of a bolus injection of epinephrine $(0.005 \mathrm{mg} / \mathrm{kg})$ and sodium bicarbonate $(1 \mathrm{mg} / \mathrm{kg}), \mathrm{CPR}$ was performed with an rodent CPR machine (Jeung Do Bio \& Plant Co., Ltd.) on the chest of the rat at the depth of one-third of the anteroposterior region with mechanical chest compression at the rate of $300 / \mathrm{min}$ to provide $100 \%$ oxygen supply until the MAP became $60 \mathrm{mmHg}$ (29). ECG was monitored continuously and the temperature was maintained according to the experimental protocol (Fig. 1A).

Temperature management among the groups. The body temperature of the Normo. group was maintained during and after surgery at $37 \pm 0.5^{\circ} \mathrm{C}$ and this temperature was further maintained until the rats were sacrificed. In the $2 \mathrm{~h}$ of $\mathrm{TH}$ group, CA was performed at a normal temperature $\left(37 \pm 0.5^{\circ} \mathrm{C}\right)$ and then the body temperature was maintained at $33 \pm 0.5^{\circ} \mathrm{C}$ immediately after CPR and the same temperature was maintained for $2 \mathrm{~h}$, following which rapid rewarming was performed with the heating pad until the normal temperature $\left(37 \pm 0.5^{\circ} \mathrm{C}\right)$ was achieved and the rats were returned to their cages until they were sacrificed (Fig. S1). In the 4 and $6 \mathrm{~h}$ of TH groups, the duration of maintaining the body temperature at $33 \pm 0.5^{\circ} \mathrm{C}$ CA after CPR was extended to 4 and $6 \mathrm{~h}$, respectively. The body temperature was monitored by a rectal temperature sensor (30). After $24 \mathrm{~h}$ of CPR, all rats were sacrificed $(13,31)$.

Detection of blood urea nitrogen (BUN) and serum creatinine $(\mathrm{Cr})$. All of the rats were anesthetized with $30 \%$ urethane $(1,400 \mathrm{mg} / \mathrm{kg}$, intraperitoneal) at $24 \mathrm{~h}$ after ROSC and transcardially perfused with $4 \%$ paraformaldehyde and $5 \mathrm{ml}$ of blood was collected from the inferior vena cava. The blood was centrifuged at $1,413 \mathrm{x}$ g for $15 \mathrm{~min}$ at $4^{\circ} \mathrm{C}$ and serum was obtained for the determination of $\mathrm{BUN}$ and $\mathrm{Cr}$ with an Olympus AU 2700 Analyzer (Olympus Optical Co., Ltd.).

Measurement of malondialdehyde (MDA) content of renal tissues. The MDA concentration in renal tissues was measured according to a previously published protocol by our group (32). In short, homogenization and centrifugation of the renal tissues were performed at $8,832 \times \mathrm{g}$ for $10 \mathrm{~min}$ at $4^{\circ} \mathrm{C}$ and 
A
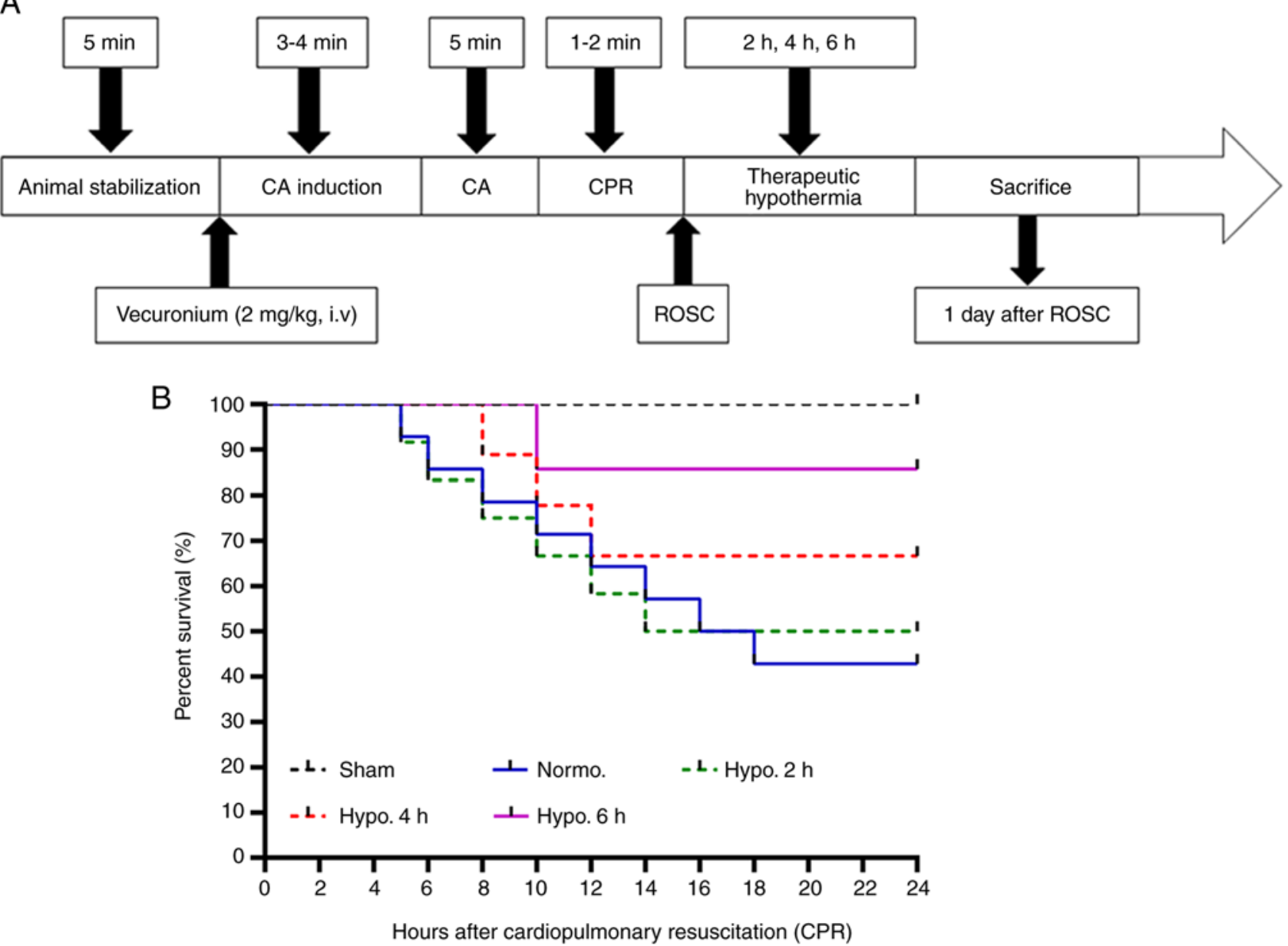

Figure 1. Schematic diagram and survival rate. (A) Schematic representation of the asphyxial CA model in rats and measurements obtained during the animal stabilization period (baseline), induction of CA, CA, CPR time, ROSC, TH duration and time-point of sacrifice. (B) The survival rate of the experimental rats was compared using Kaplan-Meier analysis ( $\mathrm{P}<0.05)$. The Normo. group had a survival rate of $42.8 \%$ and it increased significantly at $2 \mathrm{~h}(50 \%), 4 \mathrm{~h}(66.6 \%)$ and $6 \mathrm{~h}(85.7 \%)$ of TH after CPR. Groups: Normo., Normothermia + CA; Hypo., TH after ROSC following CA. CPR, cardiopulmonary resuscitation; CA, cardiac arrest; $\mathrm{TH}$, therapeutic hypothermia; ROSC, return of spontaneous circulation.

the supernatant was collected and stored at $-80^{\circ} \mathrm{C}$ for MDA analysis. Subsequently, the MDA content of the renal tissues was measured according to the instructions of a commercial kit (TBARS assay kit; Cayman Chemical).

Tissue processing. For transcardial perfusion, 0.1 M PBS ( $\mathrm{pH}$ 7.4) was used following $4 \%$ paraformaldehyde in $0.1 \mathrm{M}$ phosphate buffer ( $\mathrm{pH}$ 7.4). Kidneys were harvested from each rat and fixed with $10 \%$ neutral buffered formalin. Subsequently, the kidney was sagittally cut, embedded in paraffin and sectioned at $5 \mu \mathrm{m}$.

Histopathological changes. H\&E staining was performed to observe the histopathological changes in the kidney according to the published protocol (33). The kidney sections were mounted on glass slides, dehydrated with ethanol after staining with H\&E and mounted with Canada balsam (Kanto Chemical). Periodic acid Schiff (PAS) staining was performed according to the kit (cat. no. PAS-1-IFU; ScyTek Laboratories, Inc.) manufacturer's protocol. A Leica DM 2500 microscope (Leica Microsystems) was used to image the sections at fixed magnifications of x400 (H\&E) and x1,000 (PAS), and in each group, 10 specific areas were captured. Analysis of glomerular lesions was performed according to a previously published procedure (25). The scoring was as follows: Normal, 0; $<25 \%$ damage, $1 ; 26-50 \%$ damage, 2 ; $51-75 \%$ damage, 3 ; and $76-100 \%$ damage, 4 (25). Histopathological analysis of lesions was performed according to a published procedure (34). In short, histopathological changes were evaluated by quantitative measurement of interstitial tubular injury and through counting the number of apoptotic and necrotic cells, tubular brush border loss, dilatation of tubules, cast formation and infiltration of neutrophils. The injury scoring was as follows: 0, None; 1, 0-10; 2, 11-25; 3, 26-45; 4, 46-75; and 5, 76-100\% (34).

Immunohistochemistry (IHC). IHC analysis of $\mathrm{Nrf} 2 / \mathrm{HO}-1$ was performed according to a previously published protocol by our group (32). In short, deparaffinization and dehydration of the paraffin sections were performed in xylene and ethanol. Antigen retrieval was performed with citrate buffer and 3\% hydrogen peroxide was used for inactivation of endogenous peroxidase activity. Goat serum (cat. no. S-1000-20; Vector Laboratories, Inc.) was used for blocking of the tissue, followed by incubation with anti-rabbit polyvalent Nrf2 (cat. no. BP1-32822; Novusbio) and HO-1 (cat. no. ab13243; Abcam) antibody (dilution, 1:500). 
Table I. Physiological variables of rats after asphyxial CA.

\begin{tabular}{|c|c|c|c|c|c|}
\hline Physiological variable & Baseline & Normo. & Hypo. 2 h & Hypo. 4 h & Нyро. $6 \mathrm{~h}$ \\
\hline Body weight, g & $281 \pm 14.21$ & $286 \pm 14.51$ & $283 \pm 9.91$ & $275 \pm 20.39$ & $279 \pm 15.01$ \\
\hline Asphyxial time to CA, sec & N.A. & $139 \pm 34.68$ & $145 \pm 23.71$ & $156 \pm 31.41$ & $149 \pm 24.84$ \\
\hline CPR time, sec & N.A. & $68 \pm 10.89$ & $63 \pm 15.67$ & $75 \pm 7.85$ & $73 \pm 11.12$ \\
\hline Heart rate, beats/min & $331 \pm 10.91$ & $339 \pm 19.54$ & $335 \pm 18.74$ & $338 \pm 10.31$ & $333 \pm 8.79$ \\
\hline Room temperature, ${ }^{\circ} \mathrm{C}$ & N.A. & $24 \pm 0.70$ & $25 \pm 0.49$ & $25 \pm 0.57$ & $24 \pm 0.81$ \\
\hline
\end{tabular}

Groups: Normo., Normothermia + CA; Hypo, TH after return of spontaneous circulation following CA. TH, therapeutic hypothermia; $\mathrm{CA}$, cardiac arrest; CPR, cardiopulmonary resuscitation.

Subsequently, sections were incubated with the biotinylated secondary antibody (dilution, 1:250; cat. no. BA-4000-1.5) and Vectastain ABC reagent (cat. no. PK-4000) (both Vector Laboratories, Inc.) at room temperature for $1 \mathrm{~h}$. Diaminobenzidine was applied to the sections in the dark until the development of a brown color. After counterstaining with hematoxylin staining for 2-3 sec at room temperature, the sections were dehydrated and cleared in ethanol and xylene and then mounted on a glass slide. A Leica DM 2500 microscope (Leica Microsystems) was used to image the sections at a fixed magnification of $x 400$. From each group, 10 specific areas were captured. ImageJ threshold analysis software version $1.52 \mathrm{a}$ (National Institutes of Health) was used to measure the relative optical density (ROD\%).

Statistical analysis. GraphPad Prism 5.0 (GraphPad Software, Inc.) was used to analyze the data. Values are expressed as the mean \pm standard error or the mean. The survival rate of the rats was analyzed using Kaplan-Meier curves and log-rank tests. Furthermore, values were expressed as the median with interquartile range and statistical comparisons were performed using the Kruskal Wallis test followed by Dunn's post-hoc test. $\mathrm{P}<0.05$ was considered to indicate statistical significance.

\section{Results}

Physiological variables of the rats. The physiological parameters were insignificant between the Sham and Normo. groups $(\mathrm{P}>0.05)$. There was also no significant difference between the Normo. group and the groups with 2, 4 and 6 h of TH $(\mathrm{P}>0.05$; Table I). CA was confirmed with isoelectric ECG and $\mathrm{SpO}_{2}$ (Table I).

Survival rate. The survival rate of the rats was determined at 1 day post-CA. Kaplan-Meier analysis demonstrated a significant difference in the survival rate $(\mathrm{P}<0.05)$. In the sham group, the survival rate was $100 \%$; however, the survival rate in the Normo. group was $42.8 \%$ at 1 day after ROSC. The survival rate of rats increased significantly in the groups with $2 \mathrm{~h}(50 \%), 4 \mathrm{~h}(66.6 \%)$ and $6 \mathrm{~h}(85.7 \%)$ of $\mathrm{TH}$ after ROSC $(\mathrm{P}=0.0267$ treatment groups vs. Normo. group; Fig. 1B).

Serum BUN and Cr. Serum BUN and Cr levels, two major indexes of renal function, were significantly increased in the
Normo. group as compared with those in the sham group $(\mathrm{P}=0.0018$ and 0.0036 , respectively). After $2 \mathrm{~h}(\mathrm{P}>0.05)$ and $4 \mathrm{~h}(\mathrm{P}>0.05)$, the levels of BUN and $\mathrm{Cr}$ decreased when compared with those in the Normo. group but the change was not significant. However, BUN and $\mathrm{Cr}$ significantly decreased at $6 \mathrm{~h}$ of $\mathrm{TH}$ as compared to the Normo. group $(\mathrm{P}=0.0068$ and 0.0189, respectively; Fig. 2A and B).

MDA levels in renal tissues. MDA, a final product of lipid peroxidation, which is induced by ROS production, was measured in renal tissues with an MDA kit (13). The MDA concentration significantly increased $(\mathrm{P}=0.0338)$ in the Normo. group compared with that in the sham group. However, its level was significantly decreased in the $6 \mathrm{~h}(\mathrm{P}=0.0450)$ of $\mathrm{TH}$ groups. Its level also decreased in the $2 \mathrm{~h}(\mathrm{P}>0.05)$ and $4 \mathrm{~h}$ (P>0.05) of TH group but not significantly compared with that in the Normo. group (Fig. 2C).

Histopathological damage. Renal histopathological changes were evaluated by H\&E and PAS staining (Figs. 3 and 4). No histopathological changes were present in the kidneys of the Sham group (34). Kidney lesions were markedly increased in the Normo. group compared with those in the Sham group $(\mathrm{P}<0.0001)$. Histopathological damage was significantly decreased $(\mathrm{P}=0.0046)$ in the group with $6 \mathrm{~h}$ of $\mathrm{TH}$; however, it was not significantly decreased in the groups with $2 \mathrm{~h}$ $(\mathrm{P}>0.05)$ and $4 \mathrm{~h}(\mathrm{P}>0.05)$ of $\mathrm{TH}$ when compared with the Normo. group. The brush border of renal tubular epithelial cells was severely eroded. Tubular dilatation and acute renal tubular necrosis were more evident in the Normo. group when compared to the groups with 2, 4 and $6 \mathrm{~h}$ of TH. In addition, dilatation of glomerular capillaries was severe in the Normo. group ( $\mathrm{P}=0.0064)$ as compared to the sham group; however, it was attenuated after $2 \mathrm{~h}(\mathrm{P}>0.05), 4 \mathrm{~h}(\mathrm{P}>0.05)$ and $6 \mathrm{~h}$ $(\mathrm{P}=0.0460)$ of TH (Figs. 3 and 4$)$.

Expression of $\mathrm{Nrf} 2$ and $\mathrm{HO}-1$. IHC was performed to investigate the mechanisms of the renoprotective effects of $\mathrm{TH}$ in each group. The expression of Nrf2 and HO-1 increased in the Normo. group compared to the sham group but without any statistical significance $(\mathrm{P}>0.05)$. The ROD\% of Nrf2 expression increased insignificantly at $2 \mathrm{~h}(\mathrm{P}>0.05)$ and $4 \mathrm{~h}(\mathrm{P}>0.05)$, and was significantly increased at $6 \mathrm{~h}$ of $\mathrm{TH}(\mathrm{P}=0.0002$; Fig. 5). Furthermore, HO-1 expression was significantly increased after $6 \mathrm{~h}(\mathrm{P}=0.007)$ of $\mathrm{TH}$ but not significantly after 

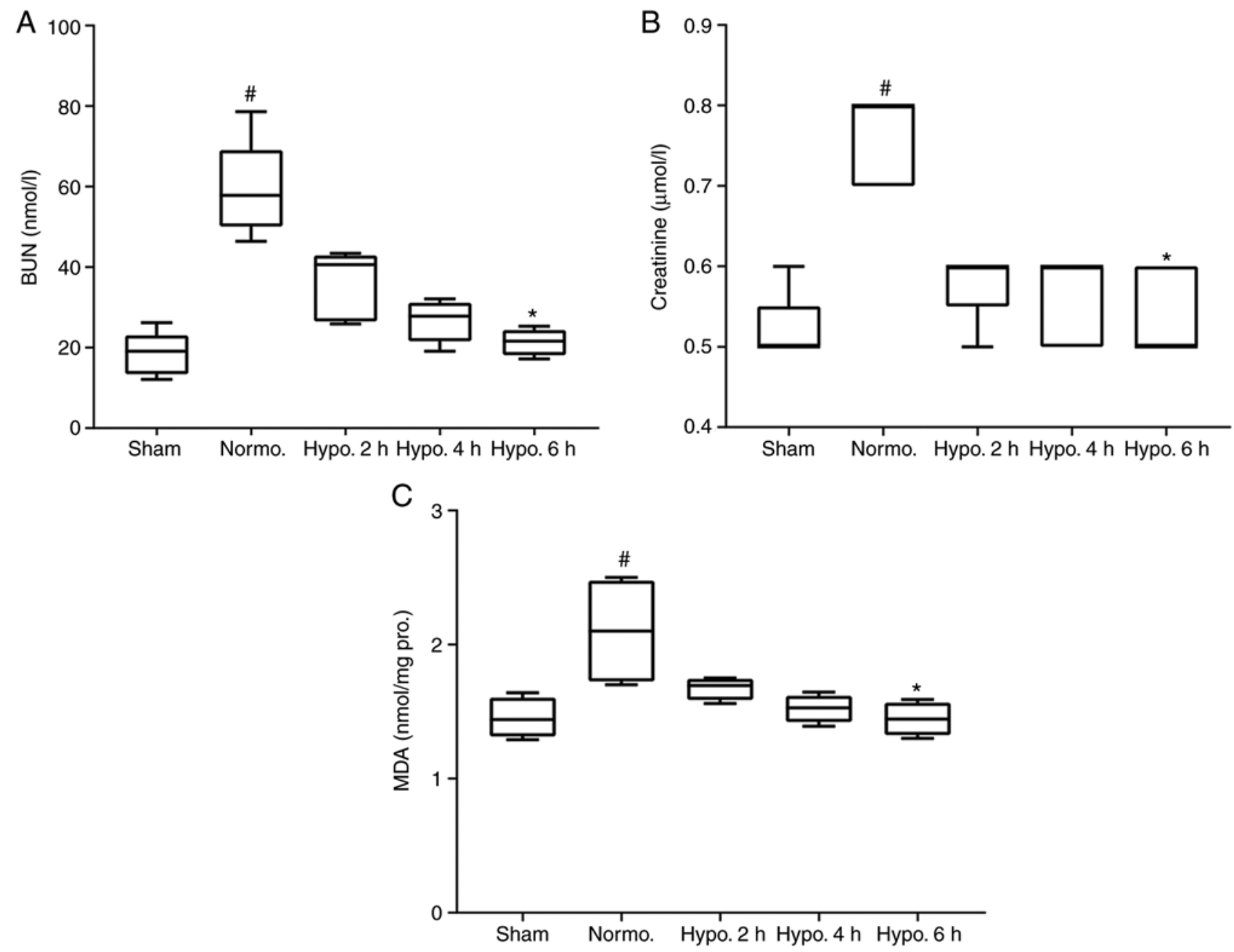

Figure 2. Serum BUN, Cr and MDA. Serum BUN, serum Cr and MDA content of the renal tissues increased significantly in the Normo. group compared to the sham group. The level of (A) BUN, (B) serum Cr and (C) MDA decreased insignificantly after 2 and $4 \mathrm{~h}$ of TH and increased significantly after $6 \mathrm{~h}$ of TH. The level of BUN, $\mathrm{Cr}$ and MDA data are presented as median and interquartile range. ${ }^{*} \mathrm{P}<0.05$ compared with the Sham group; $\mathrm{P}<0.05$ compared with the Normo. group. Groups: Normo., Normothermia + CA; Hypo., TH after return of spontaneous circulation following CA. BUN, blood urea nitrogen; Cr, creatinine; MDA, malondialdehyde; TH, therapeutic hypothermia; CA, cardiac arrest; pro., protein.

$2 \mathrm{~h}(\mathrm{P}=0.079)$ and $4 \mathrm{~h}(\mathrm{P}=0.1089)$ of $\mathrm{TH}$ when compared with the Normo. group (Fig. 6).

\section{Discussion}

The present study demonstrated that $\mathrm{TH}$ decreased the levels of BUN, serum $\mathrm{Cr}$ and MDA in renal tissues and increased the expression of Nrf2 and HO-1 in a TH time-dependent manner. The results suggested that $\mathrm{TH}$ ameliorated asphyxial CA-induced ischemia/reperfusion (I/R) renal dysfunction and oxidative stress. In the present asphyxial CA model, TH improved the survival rate and attenuated histopathological damage following ROSC after CA compared to those in the Normo. group. It is thus indicated that TH effectively mitigates oxidative stress markers in renal injury and increases the survival rate in a $\mathrm{TH}$ treatment time-dependent manner.

$\mathrm{I} / \mathrm{R}$ injury is defined as paradoxical aggravation of cellular damage and death after the restoration of blood flow to previously viable ischemic tissue (35). CA is a case of whole-body I/R injury following ROSC characterized by multi-organ dysfunction (3) and acute kidney failure is an outcome of whole-body I/R injury (24). In the present study, acute renal tubular necrosis, proximal convoluted tubule, brush border erosion and dilatation of renal glomerular capillaries were more severe in the Normo. group compared to those receiving 2, 4 and $6 \mathrm{~h}$ of $\mathrm{TH}$ after $\mathrm{CA}$. Thus, it was indicated that $\mathrm{TH}$ treatment decreased renal injury and dysfunction in a $\mathrm{TH}$ treatment time-dependent manner.

The application of hypothermia treatment is a controversial topic. In one study, it achieved no beneficial effects on the survival of patients with CA following ROSC (23). Previous studies demonstrated that the survival rate of rats reached up to 7-8\% at 2 days following ROSC after CPR in an asphyxial CA model $(33,36)$. A study on ventricular fibrillation CA also revealed a low survival rate at $72 \mathrm{~h}$ following ROSC in rats (37). In the present study, the survival rate of the rats in the Normo. group also decreased, which was similar to previous results obtained with the asphyxial CA model $(33,36,37)$. However, the present study reported that $\mathrm{TH}$ treatment increased the survival rate of the rats in a $\mathrm{TH}$ treatment time-dependent manner (23). Roberts et al (3) 

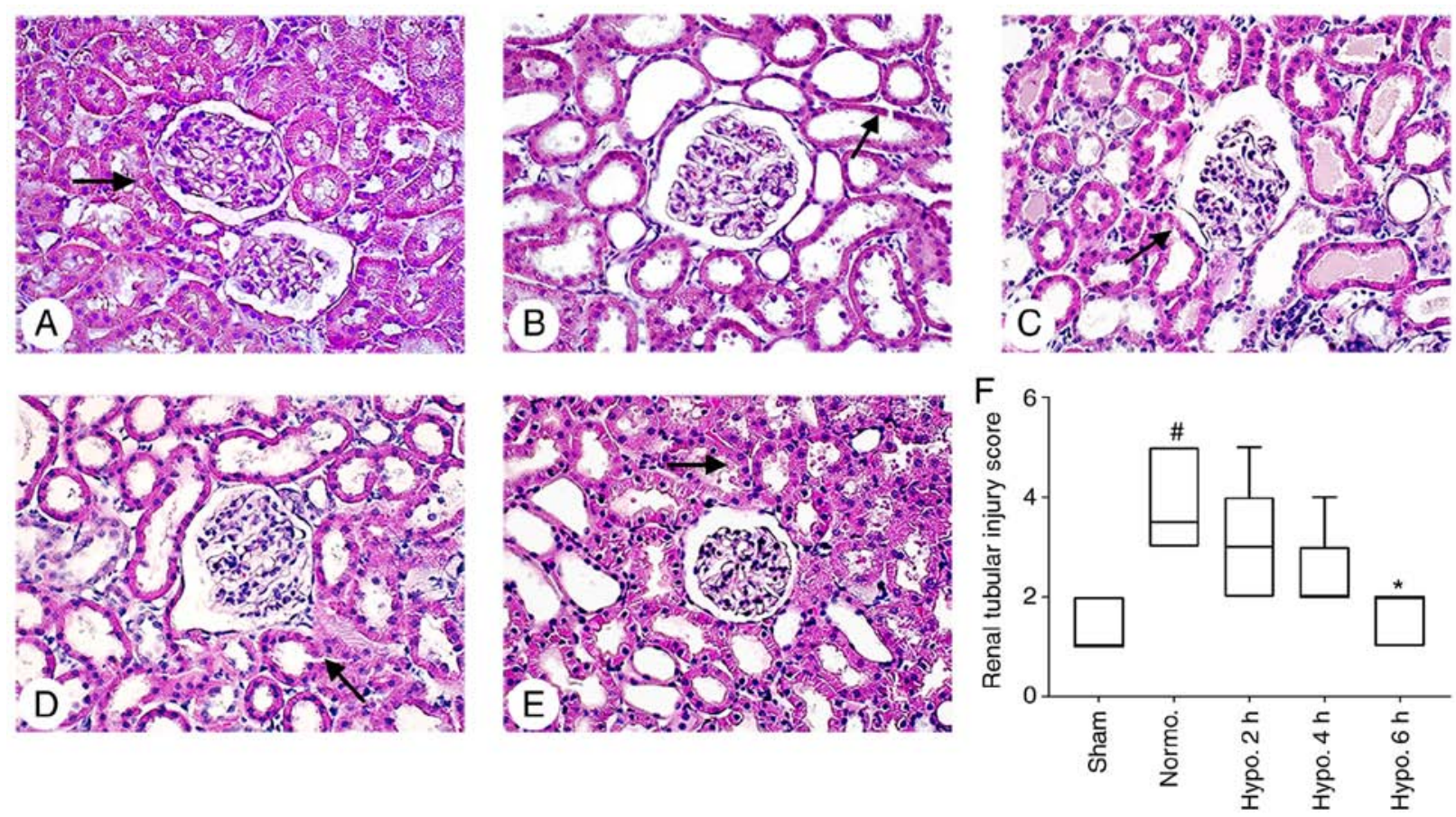

Figure 3. Histology of renal tissues in the different groups. H\&E staining of (A) the sham group indicated no tubular injury. (B) In the Normo. group, obvious tubular injury was present (arrow). Renal tubules exhibited severe dilatation and loss of brush borders with necrosis. (C-E) TH ameliorated tubular injury (arrows); (C) $2 \mathrm{~h}$, (D) $4 \mathrm{~h}$ and (E) $6 \mathrm{~h}$ of TH (magnification, $\mathrm{x} 400$ ). (F) Tubular injury score in the different groups. Glomerular tubular injury data are expressed as the median with interquartile range. ${ }^{*} \mathrm{P}<0.05$ compared with the Sham group; ${ }^{*} \mathrm{P}<0.05$ compared with the Normo. group. Groups: Normo., Normothermia + CA; Hypo., TH after return of spontaneous circulation following CA. TH, therapeutic hypothermia; CA, cardiac arrest.
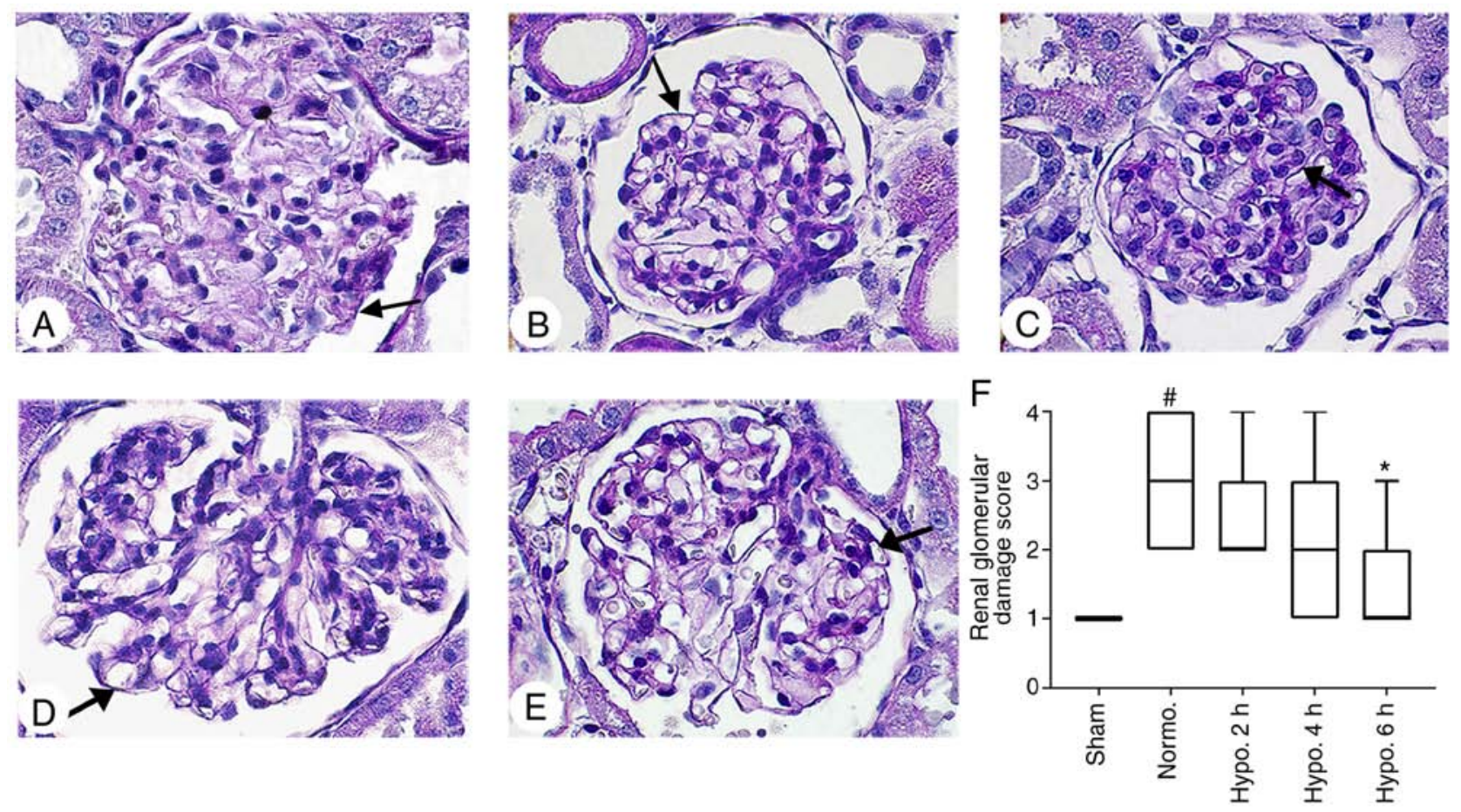

Figure 4. PAS staining. PAS staining of (A) the Sham group indicated no glomerular damage. (B) Glomerular capillary dilation (arrow) and the glomerular diameter increased significantly in the Normo. group. (C-E) Glomerular capillary dilatation was ameliorated after TH (arrows); (C) $2 \mathrm{~h}$, (D) $4 \mathrm{~h}$ and (E) $6 \mathrm{~h}$ of TH (magnification, x1,000). (F) Renal glomerular damage score in the different groups. Renal glomerular damage score data are expressed as the median with interquartile range. ${ }^{\prime} \mathrm{P}<0.05$ compared with the Sham group; " $\mathrm{P}<0.05$ compared with the Normo. group. Groups: Normo., Normothermia + CA; Hypo., TH after return of spontaneous circulation following CA. TH, therapeutic hypothermia; CA, cardiac arrest; PAS, periodic acid Schiff.

demonstrated that TH ameliorated kidney damage following ROSC; however, the injury mechanism and attenuation via
TH remained elusive. In the present study, renal injury was attenuated with TH treatment in a time-dependent manner. 

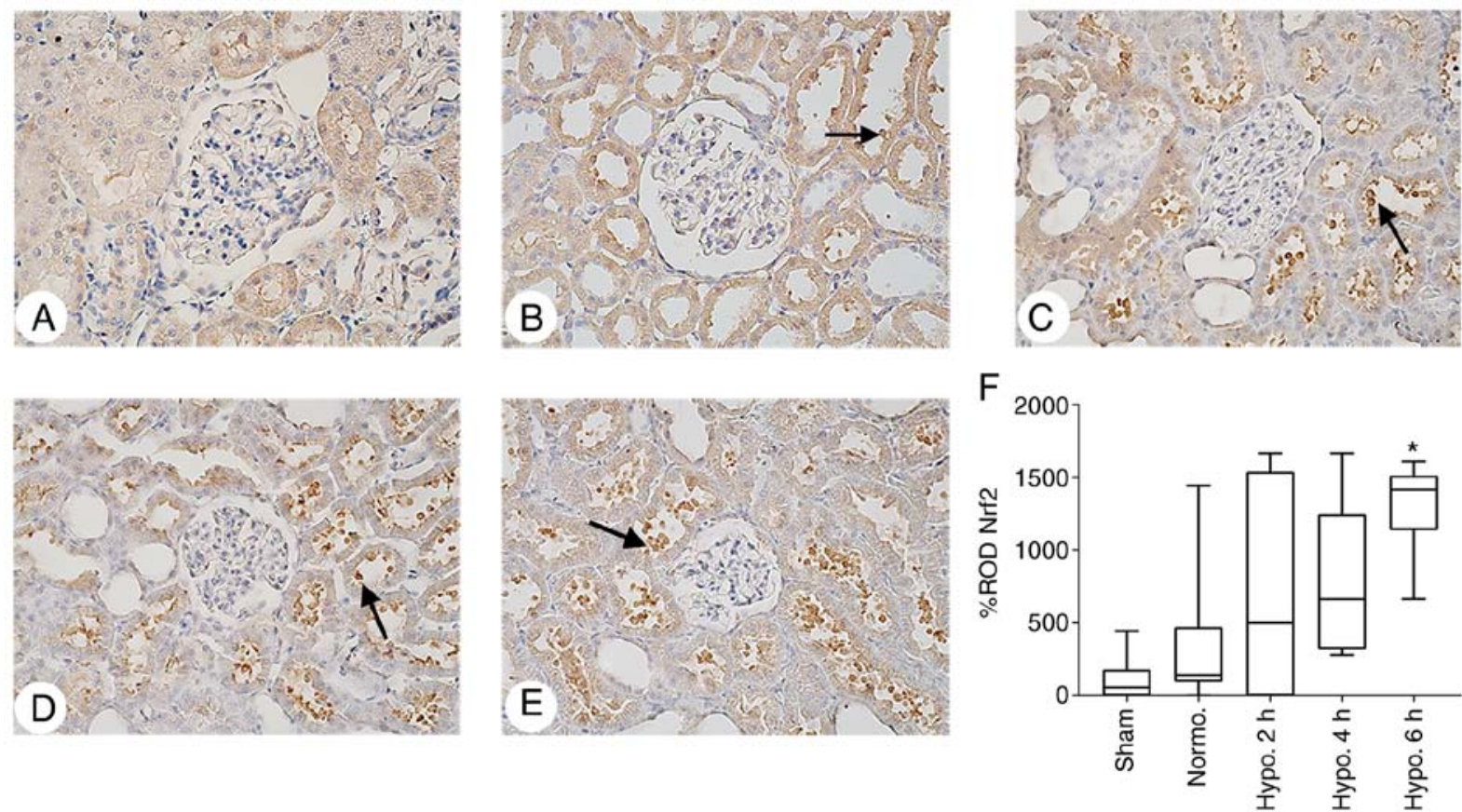

Figure 5. IHC analysis of Nrf2 expression in renal tissues. Representative IHC staining images for Nrf2 in the kidney tissues of (A) the Sham group, (B) Normo. group, (C) $2 \mathrm{~h} \mathrm{TH}$ group, (D) $4 \mathrm{~h}$ TH group and (E) $6 \mathrm{~h} \mathrm{TH}$ group (positive staining indicated by arrows; magnification, $\mathrm{x} 400$ ). It was revealed that TH increased the expression of Nrf2 in the renal cortex. (F) The ROD\% of Nrf2 expression was significantly increased at $6 \mathrm{~h}$ of TH. ROD\% of Nrf2 data are expressed as the median with interquartile range. "P $<0.05$ compared with the Normo. group. Groups: Normo., Normothermia + CA; Hypo., TH after return of spontaneous circulation following CA. TH, therapeutic hypothermia; CA, cardiac arrest; Nrf2, nuclear erythroid-related factor-2; IHC, immunohistochemical; ROD, relative optical density.
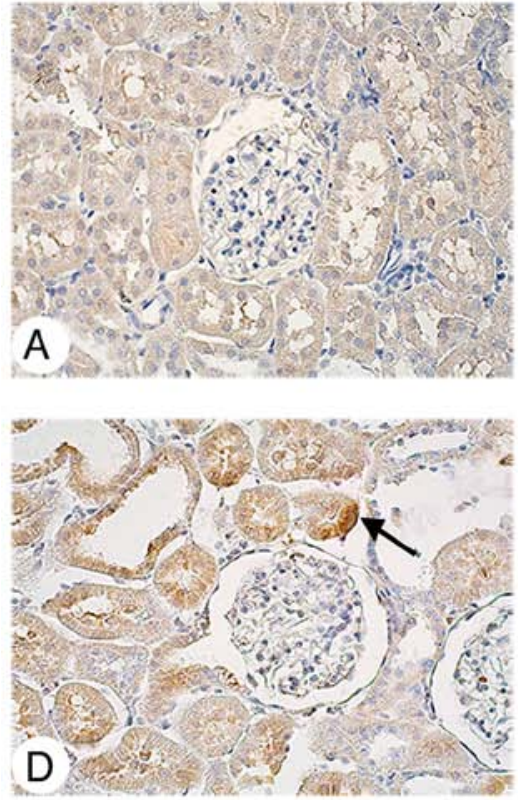
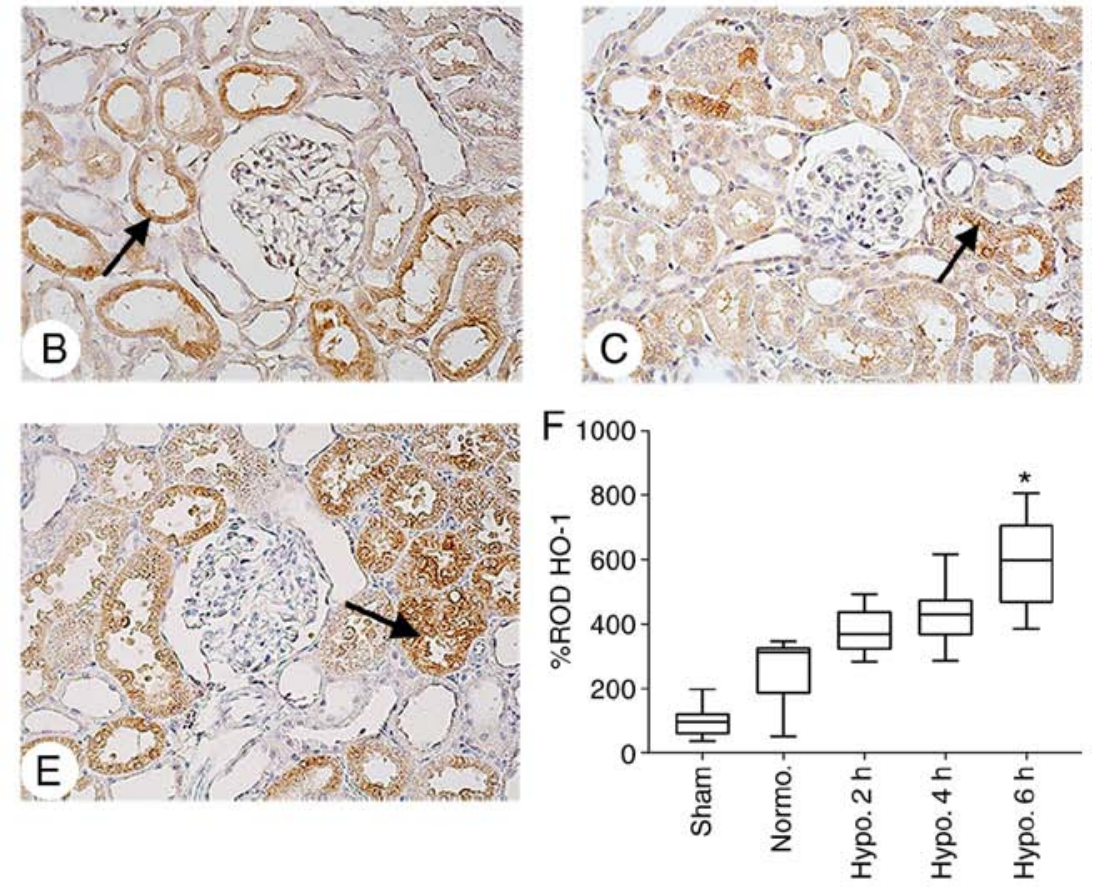

Figure 6. IHC analysis of HO-1 expression in renal cortical tissues. Representative IHC staining images for HO-1 in the kidney tissues of (A) the Sham group, (B) Normo. group, (C) $2 \mathrm{~h} \mathrm{TH}$ group, (D) $4 \mathrm{~h} \mathrm{TH}$ group and (E) $6 \mathrm{~h}$ TH group (positive staining indicated by arrows; magnification, $\mathrm{x} 400$ ). It was indicated that TH increased HO-1 expression in the renal cortical tissues. (F) The ROD\% of HO-1 staining was elevated in the Normo. vs. Sham group and increased significantly at $6 \mathrm{~h}$ of TH in comparison with the Normo. group. ROD\% of HO-1 data are expressed as the median with interquartile range. ${ }^{*}<0.05$ compared with the Normo. group. Groups: Normo., Normothermia + CA; Hypo., TH after return of spontaneous circulation following CA. TH, therapeutic hypothermia; CA, cardiac arrest; IHC, immunohistochemical; ROD, relative optical density; HO-1, heme oxygenase.

The previous and present results suggested that $\mathrm{TH}$ treatment for various durations after ROSC is a favorable factor for patients with CA and renal injury and may result in improved outcomes (3). 
Atypical or unnecessary ROS is involved in the pathogenesis of tissue damage and injury (38). Guidet and Shah (39) reported that the capacity of the kidney to produce ROS is not accompanied by a related defense system against the resulting harm. A marked level of the pro-oxidation production MDA is generated due to reaction of ROS with components of cell membranes, and Grekas et al (40) suggested that I/R injury significantly elevated MDA production in renal tissues. Furthermore, Xia et al (41) reported that mild hypothermia decreased the MDA level in the kidneys of RI/RI mice. Hackenhaar et al (42) reported that $\mathrm{TH}$ reduces serum MDA concentration following ROSC after CA. In addition, Islam et al (25) reported that $\mathrm{TH}$ decreased the level of MDA in the renal tissues of their asphyxial CA rat model. In the present study, MDA levels in the renal cortical tissues following ROSC after asphyxial CA in rats were investigated, revealing that $\mathrm{TH}$ ameliorated MDA levels in the kidneys in a TH treatment time-dependent manner, which was in agreement with other studies. In the present study, TH ameliorated renal injury time-dependently and may be associated with an increased survival rate.

Previous studies demonstrated that the levels of kidney injury markers, MDA levels, renal histopathological ailments and Nrf2/HO-1 expression levels increased at $24 \mathrm{~h}$ following $45 \mathrm{~min}$ of RI/RI; however, in the present study, asphyxial CA induced 5 min of whole-body I/R injury $(13,31)$. Despite the difference in ischemia duration and experimental models, the present study demonstrated consistency with the previous $\mathrm{RI} / \mathrm{RI}$ rat models $(13,31)$. Thus, it is suggested that renal injury markers, MDA and renal histopathological changes were attenuated and high expression level of $\mathrm{Nrf} 2 / \mathrm{HO}-1$ was achieved at $24 \mathrm{~h}$ after return of spontaneous circulation. Signaling pathways associated with asphyxial CA-induced $\mathrm{RI} / \mathrm{RI}$ remain to be fully elucidated. In the Nrf2 signaling pathway, under normal physiological conditions, Nrf2 binds with Kelch-like ECH-associated protein in the cytoplasm (43). However, the oxidative stress in pathological conditions may lead to dissociation of the Nrf2-Keap1 complex $(12,44)$, allowing Nrf2 to translocate into the nucleus, where it binds with ARE and HO-1 and results in an offset of cellular oxidative stress $(12,44)$. The Nrf2/HO-1 expression level was correlated with scavenging and amelioration of ROS during the oxidative stress process (45). Therefore, Nrf2/HO-1 expression was considered beneficial in the case of RI/RI (45). Xia and Zhang (46) demonstrated that mild hypothermia significantly upregulated the expression of $\mathrm{Nrf} 2 / \mathrm{HO}-1$ in the cerebral cortex and hippocampus following asphyxial CA of rats; however, the role of mild $\mathrm{TH}$ on $\mathrm{Nrf} 2$ and $\mathrm{HO}-1$ expression in asphyxial CA-induced-renal ischemia has remained elusive. In the present study, Nrf2 and HO-1 expression increased in the Normo. group compared with that in the sham group; however, application of TH for 2, 4 and $6 \mathrm{~h}$ increased the expression of Nrf2/HO-1 in the renal cortical tissues in a $\mathrm{TH}$ treatment time-dependent manner. Thus, it was indicated that immediate TH after CPR decreased the renal oxidative stress effects of post cardiac arrest syndrome, which is associated with post cardiac arrest myocardial dysfunction, brain injury and systemic ischemia/reperfusion response.

However, the present study still has certain limitations. TH treatment was followed by gradual rewarming in previous studies on the asphyxial CA and CPR model, in which the focus was on the brain and myocardium and the results demonstrated improved neurological outcome and myocardial function $(29,47)$. By contrast, rapid rewarming after $\mathrm{TH}$ treatment was reported to improve the survival rate, histopathological ailments and renal injury markers in the asphyxial CA rat model $(29,47)$. In the present study rapid rewarming was performed after therapeutic hypothermia $(2,4$ and $6 \mathrm{~h})$ which was one of the potential limitations and requires further investigation. Furthermore, western blot analysis of Nrf2 and HO-1 expression, use of Nrf2 and HO-1 inhibitors/agonists, the lack of an alternative method for oxidative stress measurement and the lack of a variety of different experiments to verify the results are potential limitations of the present study and an objective of future studies by our group.

In conclusion, in the present study, immediate $\mathrm{TH}$ exhibited renoprotective effects against asphyxial CA-induced RI/RI by inhibiting oxidative stress and increased the survival rate of rats in a $\mathrm{TH}$ treatment time-dependent manner. It was indicated that $\mathrm{TH}$ confers protection by decreasing ROS levels, increasing the expression levels of Nrf2 and HO-1 in renal tissues and improving the survival rate in a $\mathrm{TH}$ treatment time-dependent manner. In short, immediate TH may be a favorable protective method for asphyxial CA-induced RI/RI; however, its role in the relationship among the renal system, heart and brain in $\mathrm{CA}$ remains elusive. Further study is required to evaluate the underlying protective mechanisms of $\mathrm{TH}$ in the kidneys via asphyxial CA-induced RI/RI in rats.

\section{Acknowledgements}

Not applicable.

\section{Funding}

This research was supported by the Basic Science Research Program through the National Research Foundation of Korea funded by the Ministry of Education (grant nos. NRF-2020R1I1A3070874, NRF-2019R1C1C1002564, NRF-2019R1F1A1062696 and 2019R1A6A1A03033084) and the Biomedical Research Institute of Jeonbuk National University Hospital.

\section{Availability of data and materials}

The datasets used and/or analyzed during the current study are available from the corresponding author on reasonable request.

\section{Authors' contributions}

AJ, YJY, JHL and HJT were responsible for the experimental design, data acquisition, data analysis and manuscript writing. JHC, WST, HYS, SEK, MSI, EYL and KHK performed the experiments and data analysis. DCA, BYP, JCY and ISK performed data analyses and made critical comments on the entire process of the study. All of the authors read and approved the final version of manuscript. JHL and HJT confirm the authenticity of the raw data. 


\section{Ethics approval and consent to participate}

All the procedures were performed in full compliance with the recommendations in the guidelines of the Institutional Animal Care and Use Committee of Jeonbuk National University (approval no. JBNU 2019-005).

\section{Patient consent for publication}

Not applicable.

\section{Competing interests}

The authors declare that they have no competing interests.

\section{References}

1. Girotra S, Chan PS and Bradley SM: Post-resuscitation care following out-of-hospital and in-hospital cardiac arrest Heart 101: 1943-1949, 2015.

2. Forman-Hoffman VL, Ault KL, Anderson WL, Weiner JM, Stevens A, Campbell VA and Armour BS: Disability status, mortality, and leading causes of death in the United States community population. Med Care 53: 346-354, 2015.

3. Roberts BW, Kilgannon JH, Chansky ME, Mittal N, Wooden J, Parrillo JE and Trzeciak S: Multiple organ dysfunction after return of spontaneous circulation in postcardiac arrest syndrome. Crit Care Med 41: 1492-1501, 2013.

4. Damman K, Valente MA, Voors AA, O'Connor CM, van Veldhuisen DJ and Hillege HL: Renal impairment, worsening renal function, and outcome in patients with heart failure: An updated meta-analysis. Eur Heart J 35: 455-469, 2014.

5. Hasper D, von Haehling S, Storm C, Jörres A and Schefold JC: Changes in serum creatinine in the first 24 hours after cardiac arrest indicate prognosis: An observational cohort study. Crit Care 13: R168, 2009.

6. Geri G, Guillemet L, Dumas F, Charpentier J, Antona M, Lemiale V, Bougouin W, Lamhaut L, Mira JP, Vinsonneau C and Cariou A: Acute kidney injury after out-of-hospital cardiac arrest: Risk factors and prognosis in a large cohort. Intensive Care Med 41: 1273-1280, 2015.

7. Laurent I, Monchi M, Chiche JD, Joly LM, Spaulding C, Bourgeois B, Cariou A, Rozenberg A, Carli P, Weber S and Dhainaut JF: Reversible myocardial dysfunction in survivors of out-of-hospital cardiac arrest. J Am Coll Cardiol 40: 2110-2116, 2002.

8. Madl C and Holzer M: Brain function after resuscitation from cardiac arrest. Curr Opin Crit Care 10: 213-217, 2004.

9. Nath KA and Norby SM: Reactive oxygen species and acute renal failure. Am J Med 109: 665-678, 2000

10. Tsuda H, Kawada N, Kaimori JY, Kitamura H, Moriyama T, Rakugi H, Takahara S and Isaka Y: Febuxostat suppressed renal ischemia-reperfusion injury via reduced oxidative stress. Biochem Biophys Res Commun 427: 266-272, 2012.

11. Feng L, Ke N, Cheng F, Guo Y, Li S, Li Q and Li Y: The protective mechanism of ligustrazine against renal ischemia/reperfusion injury. J Surg Res 166: 298-305, 2011.

12. Jaiswal AK: Nrf2 signaling in coordinated activation of antioxidant gene expression. Free Radic Biol Med 36: 1199-1207, 2004

13. Jiang G, Liu X, Wang M, Chen H, Chen Z and Qiu T: Oxymatrine ameliorates renal ischemia-reperfusion injury from oxidative stress through Nrf2/HO-1 pathway. Acta Cir Bras 30: 422-429, 2015.

14. Zhang Y, Rong S, Feng Y, Zhao L, Hong J, Wang R and Yuan W: Simvastatin attenuates renal ischemia/reperfusion injury from oxidative stress via targeting Nrf2/HO-1 pathway. Exp Ther Med 14: 4460-4466, 2017.

15. Williams GR Jr and Spencer FC: The clinical use of hypothermia following cardiac arrest. Ann Surg 148: 462-468, 1958.

16. Palmers PJ, Hiltrop N, Ameloot K, Timmermans P, Ferdinande B, Sinnaeve P, Nieuwendijk R and Malbrain ML: From therapeutic hypothermia towards targeted temperature management: A decade of evolution. Anaesthesiol Intensive Ther 47: 156-161, 2015.
17. Hypothermia after Cardiac Arrest Study Group: Mild therapeutic hypothermia to improve the neurologic outcome after cardiac arrest. N Engl J Med 346: 549-556, 2002.

18. OstadalP,Mlcek M,Kruger A,Horakova S, Skabradova M,Holy F, Svoboda T, Belohlavek J, Hrachovina V, Taborsky L, et al: Mild therapeutic hypothermia is superior to controlled normothermia for the maintenance of blood pressure and cerebral oxygenation, prevention of organ damage and suppression of oxidative stress after cardiac arrest in a porcine model. J Transl Med 11: 124, 2013.

19. Gong P, Li CS, Hua R, Zhao H, Tang ZR, Mei X, Zhang MY and Cui J: Mild hypothermia attenuates mitochondrial oxidative stress by protecting respiratory enzymes and upregulating MnSOD in a pig model of cardiac arrest. PLoS One 7: e35313, 2012.

20. Dohi K, Miyamoto K, Fukuda K, Nakamura S, Hayashi M, Ohtaki H, Shioda S and Aruga T: Status of systemic oxidative stress during therapeutic hypothermia in patients with post-cardiac arrest syndrome. Oxid Med Cell Longev 2013: 562429,2013

21. Legriel S, Lemiale V, Schenck M, Chelly J, Laurent V, Daviaud F, Srairi M, Hamdi A, Geri G, Rossignol T, et al: Hypothermia for neuroprotection in convulsive status epilepticus. N Engl J Med 375: 2457-2467, 2016.

22. Moler FW, Silverstein FS, Holubkov R, Slomine BS, Christensen JR, Nadkarni VM, Meert KL, Browning B, Pemberton VL, Page K, et al: Therapeutic hypothermia after in-hospital cardiac arrest in children. N Engl J Med 376: 318-329, 2017.

23. Nielsen N, Wetterslev J, Cronberg T, Erlinge D, Gasche Y, Hassager C, Horn J, Hovdenes J, Kjaergaard J, Kuiper M, et al: Targeted temperature management at $33^{\circ} \mathrm{C}$ versus $36^{\circ} \mathrm{C}$ after cardiac arrest. N Engl J Med 369: 2197-2206, 2013.

24. Tujjar O, Mineo G, Dell'Anna A, Poyatos-Robles B, Donadello K, Scolletta S, Vincent JL and Taccone FS: Acute kidney injury after cardiac arrest. Crit Care 19: 169, 2015.

25. Islam A, Kim SE, Yoon JC, Jawad A, Tian W, Yoo YJ, Kim IS, Ahn D, Park BY, Hwang Y, et al: Protective effects of therapeutic hypothermia on renal injury in an asphyxial cardiac arrest rat model. J Thermal Biol 94: 102761, 2020.

26. Drabek T, Foley LM, Janata A, Stezoski J, Hitchens TK, Manole MD and Kochanek PM: Global and regional differences in cerebral blood flow after asphyxial versus ventricular fibrillation cardiac arrest in rats using ASL-MRI. Resuscitation 85: 964-971, 2014

27. Aoki T, Okuma Y, Becker LB, Hayashida K and Shinozaki K: Methodological issue of mitochondrial isolation in acute-injury rat model: Asphyxia cardiac arrest and resuscitation. Front Med (Lausanne) 8: 666735, 2021.

28. Junyun H, Hongyang L, Ruoxian D, Young L, Shanbao T and Xiaofeng J: Real-time monitoring of cerebral blood flow by laser speckle contrast imaging after cardiac arrest in rat. Annu Int Conf IEEE Eng Med Biol Soc 2015: 6971-6974, 2015.

29. Lu J, Qian HY, Liu LJ, Zhou BC, Xiao Y, Mao JN, An GY, Rui MZ, Wang T and Zhu CL: Mild hypothermia alleviates excessive autophagy and mitophagy in a rat model of asphyxial cardiac arrest. Neurol Sci 35: 1691-1699, 2014.

30. Park Y, Ahn JH, Cho JH, Tae HJ, Lee TK, Kim B, Lee JC, Park JH, Shin MC, Ohk TG, et al: Effects of hypothermia on inflammatory cytokine expression in rat liver following asphyxial cardiac arrest. Exp Ther Med 21: 626, 2021.

31. Tong $\mathrm{F}$ and Zhou X: The Nrf2/HO-1 pathway mediates the antagonist effect of L-arginine on renal ischemia/reperfusion injury in rats. Kidney Blood Press Res 42: 519-529, 2017.

32. Akanda MR, Kim IS, Ahn D, Tae HJ, Nam HH, Choo BK, Kim K and Park BY: Anti-inflammatory and gastroprotective roles of rabdosia inflexa through downregulation of pro-inflammatory cytokines and MAPK/NF-kappaB signaling pathways. Int J Mol Sci 19: 584, 2018.

33. Park Y, Tae HJ, Cho JH, Kim IS, Ohk TG, Park CW, Moon JB, Shin MC, Lee TK, Lee JC, et al: The relationship between low survival and acute increase of tumor necrosis factor $\alpha$ expression in the lung in a rat model of asphyxial cardiac arrest. Anat Cell Biol 51: 128-135, 2018.

34. Kocoglu H, Ozturk H, Ozturk H, Yilmaz F and Gulcu N: Effect of dexmedetomidine on ischemia-reperfusion injury in rat kidney: A histopathologic study. Renal Failure 31: 70-74, 2009.

35. Collard CD and Gelman S: Pathophysiology, clinical manifestations, and prevention of ischemia-reperfusion injury. Anesthesiology 94: 1133-1138, 2001. 
36. Tae HJ, Kang IJ, Lee TK, Cho JH, Lee JC, Shin MC, Kim YS, Cho JH, Kim JD, Ahn JH, et al: Neuronal injury and tumor necrosis factor-alpha immunoreactivity in the rat hippocampus in the early period of asphyxia-induced cardiac arrest under normothermia. Neural Regen Res 12: 2007-2013, 2017.

37. Janata A, Magnet IA, Schreiber KL, Wilson CD, Stezoski JP Janesko-Feldman K, Kochanek PM and Drabek T: Minocycline fails to improve neurologic and histologic outcome after ventricular fibrillation cardiac arrest in rats. World J Crit Care Med 8: 106-119, 2019.

38. McCord JM: Oxygen-derived free radicals in postischemic tissue injury. N Engl J Med 312: 159-163, 1985.

39. Guidet BR and Shah SV: In vivo generation of hydrogen peroxide by rat kidney cortex and glomeruli. Am J Physiol 256: F158-F164, 1989.

40. Grekas D, Dioudis C, Papageorgiou G, Iliadis S, Zilidis C, Alivanis P, Dimitriadou A and Tourkantonis A: Lipid peroxidation after acute renal ischemia and reperfusion in rats: The effect of trimetazidine. Ren Fail 18: 545-552, 1996.

41. Xia Z, Wang W, Xiao Q, Ye Q, Zhang X and Wang Y: Mild hypothermia protects renal function in ischemia-reperfusion kidney: An experimental study in mice. Transplant Proc 50: 3816-3821, 2018

42. Hackenhaar FS, Medeiros TM, Heemann FM, Behling CS, Putti JS, Mahl CD, Verona C, da Silva ACA, Guerra MC, Gonçalves CAS, et al: Therapeutic hypothermia reduces oxidative damage and alters antioxidant defenses after cardiac arrest. Oxid Med Cell Longev 2017: 8704352, 2017.
43. Kim HJ and Vaziri ND: Contribution of impaired Nrf2-Keap1 pathway to oxidative stress and inflammation in chronic renal failure. Am J Physiol Renal Physiol 298: F662-F671, 2010.

44. Kobayashi $M$ and Yamamoto $M$ : Molecular mechanisms activating the Nrf2-Keap1 pathway of antioxidant gene regulation. Antioxid Redox Signal 7: 385-394, 2005.

45. Zhang L, Zhu Z, Liu J, Zhu Z and Hu Z: Protective effect of $\mathrm{N}$-acetylcysteine (NAC) on renal ischemia/reperfusion injury through Nrf2 signaling pathway. J Recept Signal Transduct Res 34: 396-400, 2014.

46. Xia D and Zhang H: Effects of mild hypothermia on expression of NF-E2-related factor 2 and heme-oxygenase-1 in cerebral cortex and hippocampus after cardiopulmonary resuscitation in rats. Iran J Basic Med Sci 20: 1002-1008, 2017.

47. Lu X, Ma L, Sun S, Xu J, Zhu C and Tang W: The effects of the rate of postresuscitation rewarming following hypothermia on outcomes of cardiopulmonary resuscitation in a rat model. Crit Care Med 42: e106-113, 2014

This work is licensed under a Creative Commons Attribution-NonCommercial-NoDerivatives 4.0 International (CC BY-NC-ND 4.0) License. 